\title{
Anthropocentricisms in cladograms
}

\author{
Hanno Sandvik
}

Received: 29 June 2007 / Accepted: 5 November 2007/Published online: 11 December 2007

(C) The Author(s) 2007

\begin{abstract}
Both written and graphic accounts of history can be biased by the perspective of the historian. O'Hara (Biol Philos 7:135-160, 1992) has demonstrated that this also applies to evolutionary history and its historians, and identified four narrative devices that introduce anthropocentricisms into accounts of phylogeny. In the current paper, I identify a fifth such narrative device, viz. the left-right ordering of the taxa at the tips of cladograms. I define two measures that make it possible to quantify the degree of anthropocentricism of cladograms, the human attention score and human rightness score. I then carry out an analysis of the presence of the different distorting mechanisms in phylogenetic textbooks. I deliberately chose two textbooks that adopted a cladistic perspective, since their authors can be assumed to be more conscious about the aim of avoiding anthropocentricisms. Three of the narrative devices are thus absent from cladistic works. However, there is a weak tendency that the resolution of cladogram branches is biased in favour of Homo sapiens. Furthermore, the human perspective is clear and highly significant in the positioning of taxa along the left-right axis of cladograms. I discuss the reasons for and implications of these biased presentations.
\end{abstract}

Keywords Anthropocentricism · Anthropocentrism · Cladogram ·

Evolutionary tree $\cdot$ Phylogeny $\cdot$ Tree balance

\section{Introduction}

Just as history can be represented by fundamentally different narratives, depending on the background of the historian (i.e., her or his ethnic, social, sexual, political

\footnotetext{
H. Sandvik ( $\bowtie)$

Department of Biology, Norwegian University of Science and Technology (NTNU), 7491

Trondheim, Norway

e-mail: hanno@evol.no
} 
background etc.), the presentation of the evolutionary past is not independent of it being written down by only one species. In an excellent essay on this topic, Robert O'Hara (1992) has enumerated different "narrative devices" that implicitly introduce a human perspective into representations of the evolutionary past. The consequence of these distortions is that most biologically untrained people's understanding of the principles of evolution is severely biased.

With this paper, I intend to do two things: to add a fifth narrative device to O'Hara's list, and to examine how common the different devices are in representations of evolutionary history.

\section{Five ways to distort evolutionary history}

O'Hara (1992, pp. 144-149) described four narrative devices commonly used in both scientific and popular, graphic and written accounts of evolutionary history. These devices are (1) the sequencing of contemporary taxa, (2) the pruning of side branches, (3) the use of paraphyletic groups, and (4) the differential resolution of branches. All narrative devices distort evolutionary history, normally by favouring the perspective or position of a single species, viz. our own, Homo sapiens. Instead of our species as such, the focus may also be on larger taxa, which, in this case, use to encompass Homo sapiens as one of its subgroups. This means that even though some narratives do not even mention man, they may still be anthropocentric by favouring the perspective of primates (Primates), mammals (Mammalia), terrestrial vertebrates (Tetrapoda), vertebrates (Vertebrata), or multicellular animals (Metazoa). In these cases, the "resolution" chosen for the narrative is too coarse to mention humans specifically. In the following, I will use the term focal taxon rather than "man" because (a) the focal taxon might not be Homo sapiens but a more inclusive taxon containing this species, because (b) the methods described below are equally applicable to any taxon of interest and to questions unrelated to Homo sapiens, and because (c) I would like to avoid the androcentricism inherent in the English vernacular name of Homo sapiens.

I will now first explain O'Hara's narrative devices in some more detail, before defining a fifth device:

(1) The sequencing of contemporary taxa is the arrangement of taxa in such a way that an-often implicit—narrative axis passes through the diagram, culminating in the focal taxon, which is placed, in graphical representation, at top or, in written representations, at the end of the narrative. The mistake is, of course, that contemporary taxa cannot possibly be related to each other in a linear sequence. All contemporary taxa are endpoints of evolutionary, branching lineages, each with its own, unique evolutionary history. Sequencing may be heuristically useful for certain purposes, but clearly not for illustrating evolutionary history.

(2) The pruning of side branches is characteristic of narratives that place a certain species at the top of a row of reconstructed stem species. In writing, the same is achieved if the narrative proceeds along the stem species of the focal 
species. In both cases, other species that arose from the same stem species are ignored. This has the effect of creating "a 'main line' of evolution" (O'Hara 1992, p. 147), while all other lines are ignored or suppressed, even though they are equally real and important.

(3) Paraphyletic groups abound in certain presentations of evolutionary trees, especially as "stem groups" in phylograms. This practice subsumes in an artificial grouping all species (extinct or otherwise) that do not belong to the focal taxon. By doing so, again, more attention is apportioned to the focal taxon.

(4) Differential resolution describes the practice to collapse different parts of a tree to differing degrees. In the extreme case, one can imagine a comb-like tree with all groups except the focal taxon collapsed into one unbranched terminal taxon (Fig. 1a).

(5) The fifth narrative device, which I want to add, is the arrangement of taxa in a cladogram along the left-right axis (given a vertically drawn time axis). This narrative device may be perceived as a special case of O'Hara's first one, but he did not include branching trees into this category. Unless the topology of a branching tree is a comb (cf. device 4), the terminal taxa of a tree do not constitute a sequence. Therefore, and to emphasize the importance of left-right ordering, it seems appropriate to mention this point as a fifth narrative device.

What all these narrative devices have in common, is that they distort evolutionary history by imposing a trend on it. The devices implicitly or explicitly make one taxon the focus and end-point of the narrative. This, in turn, suggests that the evolutionary process underlying the historical account had been aiming for that focal taxon. Needless to say, this is not in accordance with modern evolutionary thought. Evolution is no longer understood as a directed process.

Consequently, the first two narrative devices are nowadays restricted to drawings and popular accounts of evolution. Although those might be the sources for most of laypeople's evolutionary knowledge (for examples, see O'Hara 1992), I will not consider them further in this analysis. A special situation is the practice, especially among molecular systematists, to display species names at the tips of branches whether or not they are each others' closest relatives (e.g., "man, rat, chicken, trout, rhubarb, yeast"). Such species are readily interpreted by fellow scientists to be representatives of larger lineages. This is not strictly speaking a case of pruning, because all species included in the study are normally displayed. Rather, this practice makes explicit which representatives have been chosen from which
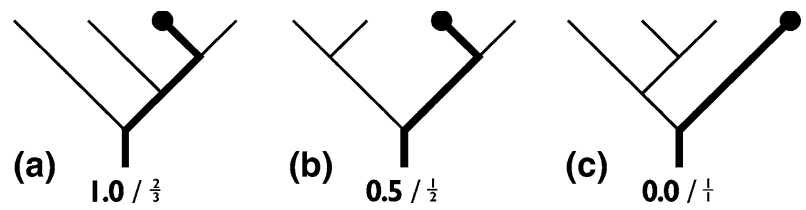

Fig. 1 Example topologies illustrating anthropocentricisms in cladograms. All cladograms have four tips (terminal taxa). The dot symbolises Homo sapiens (or another focal taxon), the bold line the path leading to the focal taxon. Below the cladograms, the human attention score (before the slash) and the human rightness score are specified (after the slash) 
lineages. I do not intend to discuss the merits or disadvantages of this practice here, and will only consider tree drawings that have not deliberately excluded certain branches.

The third device is, by definition, not found in cladograms or taxonomic systems obtained by means of cladistic analysis, i.e., in phylogenetic systematics. However, many biological textbooks are still using taxonomic groupings that are based on evolutionary systematics, so students of biology still meet this kind of distortion of the evolutionary past. In most scientific papers and books, on the other hand, taxa that are known to be paraphyletic are avoided. This reduces the prevalence of this device.

However, the fourth and fifth device can also be found in cladograms or phylogenetic systems. The remainder of this manuscript investigates the occurrence of these narrative devices in some scientific books that explicitly adopt cladistic approaches.

\section{Two numeric measures of anthropocentricism}

In order to look for the presence of narrative devices in published cladograms, one needs some sort of metric to represent the degree of anthropocentricism. I here define two such scores and accompanying significance tests, measuring the attention given to the focal taxon or its placement in a cladogram. Both scores and tests are based on the assumption that all branchings are dichotomous (bifurcating).

The human attention score aims at measuring to which degree the resolution of the branches in a cladogram favours our own species/the focal taxon. It is based on two variables, the number of tips of a cladogram, $t$; and the number of branches that are side branches to the focal taxon, $s$. The number of tips is straightforward to count. The number of side branches is identical to the number of internal nodes between the focal taxon itself (which is not counted) and the cladogram's basalmost node (which is counted). The human attention score is then calculated as

$$
\text { Human attention score }=\frac{1}{2}+\frac{1}{2} \cdot\left\{\begin{array}{l}
\text { if } s>\mathrm{lb} t:(s-\mathrm{lb} t) /(t-1-\mathrm{lb} t) \\
\text { if } s \leq \mathrm{lb} t:(s-\mathrm{lb} t) /(\mathrm{lb} t-1)
\end{array}\right.
$$

with $t>2$, number of tips; $s \in[1 ; t-1]$, number of side branches; lb, binary logarithm $\left(\log _{2}\right)$. This score has the following desirable properties:

(a) It can attain values between zero and unity, inclusively.

(b) It reaches its maximum value (unity) if and only if the cladogram is a perfectly comb-like tree where all branches are completely unresolved side branches to the focal taxon (e.g., Fig. 1a).

(c) It reaches its minimum value (zero) if and only if the focal taxon is a completely unresolved side branch to the remainder of the cladogram (e.g., Fig. 1c).

(d) It attains the value 0.5 if and only is the cladogram is perfectly balanced (e.g., Fig. 1b). 
Of course, the scaling of the score is not only convenient but also arbitrary-the score could have been scaled in many other ways. The significance test described below is not sensitive to the specific values of the score, however. Rather, it relies on whether the score of an observed cladogram is higher or lower relative to other possible topologies. The order of scores is not affected by the scaling chosen.

The significance test of the human attention score consists of calculating two numbers and their ratio. The denominator is the number of possible tree topologies that can be used to displace the interrelationships of the same taxon as in the cladogram observed, holding the root and the number of tips constant. The numerator is the number of these topologies that have the same human attention score as, or a higher human attention score than, the observed topology. In other words, the significance test asks the following question: "What is the probability that a cladogram constrained to have $t$ tips is at least as anthropocentric as the one observed, if the resolution of the cladogram's branches is randomly assigned?"

Calculation of the denominator may require some more explanation: it specifies the number of possible topologies given rooted, unlabelled and bifurcating trees with $t$ tips, and given the additional condition that topologies are counted as different even if they could be rendered congruent by rotating any number of branches around their nodes (see Felsenstein (2004) for a definition of the terms "rooted", "unlabelled" and "bifurcating"). Expressed in other words, the denominator is the number of ways the original cladogram could be modified by collapsing two (or more) sister tips into one branch, and resolving tips into two (or more) daughter branches. In this process, the total number of tips and the identity of the basalmost node (root) must not be changed. The original topology is also counted. For example, in the case of a three-tip cladogram (A-(B-C)), there is only one such alternative to the topology observed: the two sister tips (B and C) can be collapsed into one, and the third tip (A) can be shown with a higher resolution $\left(\left(\mathrm{A}_{1}-\right.\right.$ $\left.\left.\mathrm{A}_{2}\right)-\mathrm{BC}\right)$. The denominator is thus a function of the number of tips alone, where three tips result in two topologies, four tips in five topologies, and 5-12 tips in 14, 42, 132, 429, 1430, 4862, 16796 and 58786 possible topologies, respectively.

For simplicity, one might add the convention that the focal taxon always be placed at the rightmost position in all tree topologies. This assumption does not affect the outcome (in contradistinction to the rightness score described below), but it may make the counting a bit easier if done by hand.

The calculation of the human attention score is based on the assumptions that all tips that are resolved into daughter branches are in fact resolvable. This assumption is violated if some of the tips in the original cladogram are species (or species-poor taxa). In such cases, the resolution of the cladogram is constrained by the balance of the factual phylogeny (Farris 1976; Slowinski 1990; Kirkpatrick and Slatkin 1993), and not only a result of arbitrary decisions made by the scientist drawing the cladogram. I will return to an example of such a case in the Discussion.

For trees to be significantly anthropocentric according to this measure, a cladogram of at least six tips is required. A human attention score of 1 will then have a probability of 0.024 . 
To estimate the total human attention score for a number of related cladograms, the cladograms cannot simply be combined in one graph, since this can affect the attention score. To illustrate why, imagine that the information contained in one comb-like cladogram can be spread over a series of smaller cladograms with two tips each, all of which would (by definition) be perfectly balanced. The situation can therefore be addressed by averaging the scores of all cladograms. The probability must then be derived by bootstrapping: for a number of iterations, the number of side branches of each of the cladograms is randomised (again holding the number of tips of each cladogram constant). The proportion of average randomised human attention scores being equal to or higher than the one observed, is then the probability.

The human rightness score measures to which degree the focal species is placed at the right-hand side of the cladogram. It is based on the ratio $r / n$ of two counts of nodes. The denominator $n$ is the total number of nodes on the way from (and including) the basalmost one all the way up to (and excluding) the focal tip. The numerator $r$ is the number of these nodes were one has to go to the right-hand side on the way up to the focal tip. If the focal tip is the rightmost tip of the cladogram, one has to go to the right-hand side at all nodes ( $r=n$; e.g., Fig. 1c), and the human rightness score is unity. Correspondingly, if the focal tip is the leftmost tip $(r=0)$, the human rightness score is zero. A human rightness score of 0.5 is obtained if, on the way from the root to the focal taxon, one has to turn left and right equally often.

The significance test of the human rightness score consists of calculating two numbers and their ratio. The denominator is the number of possible paths one could take from the root up to the focal taxon if the number of nodes between the root and the focal tip is held constant. This number can be obtained by rotating all branches around their nodes. (Nodes that do not lie on the path from the root to the focal taxon are of course ignored, since these do not affect the outcome.) As there are two possibilities (turn right or turn left) for each node on this path, the denominator equals $2^{n}$.

The numerator is the number of these different paths that result in the same human rightness score as, or a higher human rightness score than, the path observed in the cladogram. This number can be derived from the $(n+1)$ th row of Pascal's triangle, summing its elements $r+1$ to $n+1$. For trees to be significantly anthropocentric according to this second measure, a cladogram of at least five tips is required. A human rightness score of 1 will then have a probability of 0.031 .

To estimate the total human rightness score for a number of related cladograms, those cladograms are simply combined in one large tree with unchanged topology and unchanged left-right sorting. Contrary to the human attention score, the human rightness score is not affected by the subdivision of one cladogram into several small ones. Consequently, there is no need for special adjustment in order to obtain the overall probability. It is simply calculated from the combined topology.

A possible modification of the human rightness score is to transform it to a "human utterness score" by assuming that the outermost position on the left-hand side gives equally much attention to the focal taxon as the outermost position on the right-hand side. This test can be viewed as a two-tailed version of the (one-tailed) human rightness score, and its probability is calculated accordingly. It may also be 
Table 1 Anthropocentricisms in two cladistic textbooks of animal evolution as measured by the human attention score

\begin{tabular}{lclll}
\hline Author/(volume)/figure & Number of tips & Side branches & Human attention score & Probability \\
\hline $\mathrm{Ax} / \mathrm{I} / \mathrm{I} 2$ & 8 & 6 & 0.875 & 0.016 \\
$\mathrm{Ax} / \mathrm{III} / 33$ & 7 & 6 & 1.000 & 0.0076 \\
$\mathrm{Ax} / \mathrm{III} / 68$ & 4 & 2 & 0.500 & 0.60 \\
$\mathrm{Ax} / \mathrm{III} / 74$ & 6 & 1 & 0.000 & 1.00 \\
$\mathrm{Ax} / \mathrm{III} / 84$ & 4 & 1 & 0.000 & 1.00 \\
Ax/III/89 & 8 & 3 & 0.500 & 0.38 \\
Ax/III/106 & 18 & 5 & 0.532 & 0.13 \\
Ax/unweighted mean & & & 0.487 & 0.12 \\
Ax/weighted mean & & & 0.538 & 0.016 \\
Nielsen/1.2 & 32 & 6 & 0.519 & 0.084 \\
\hline
\end{tabular}

The resolution of the cladograms' branches is weakly biased in favour of Homo sapiens

most appropriate in cases were the cladograms are arranged from top to bottom, or in other cases were it is not obvious that one should expect a left-to-right reading norm.

The two scores are illustrated in Fig. 1 with three simple cladograms consisting of four tips. Topology $1 \mathrm{~b}$ is perfectly balanced (human attention score of 0.5 ). The remaining two cladograms have comblike topologies, $1 \mathrm{a}$ in favour of the focal taxon and $1 \mathrm{c}$ in disfavour of it (human attention scores of 1 and 0 , respectively). As regards "human rightness", topology $1 \mathrm{~b}$ is the least anthropocentric (score of $1 /$ $2=0.5)$, followed by $1 \mathrm{a}(2 / 3=0.67)$ and $1 \mathrm{c}(1 / 1=1)$. None of the biases in Fig. 1 are significant because the number of tips is too small to enable a distinction between random and systematic biases. $\mathbf{R}$ functions to calculate the two scores and their probabilities are available from the author. ${ }^{1}$

\section{A case study using phylogenetic textbooks}

The algorithms developed above were applied to two textbooks in animal phylogenetics, Das System der Metazoa (Ax 1995-2001) and Animal evolution (Nielsen 2001). Nielsen summarised all relevant information in one cladogram (his Fig. 1.2). Ax, however, had subdivided the animal phylogeny into several smaller portions. Some of those cladograms overlap in the taxa displayed. In those cases, only one of the overlapping cladograms was utilised in my analysis. This was to ensure statistical independence within my sample of tree topologies.

The results of the analyses are summarised in Table 1 for the human attention score, and in Table 2 for the human rightness score.

\footnotetext{
1 The $\mathbf{R}$ functions ( $\mathbf{R}$ development core team 2005) used to calculate the human attention score, the human rightness score, and the accompanying probabilities are available on the World Wide Web at the URL http://www.evol.no/hanno/08/BiolPhil.htm.
} 
Table 2 Anthropocentricisms in two cladistic textbooks of animal evolution as measured by the human rightness score

\begin{tabular}{lcclll}
\hline Author/(volume)/figure & $\begin{array}{l}\text { Number of } \\
\text { nodes }\end{array}$ & $\begin{array}{l}\text { Human } \\
\text { node }\end{array}$ & $\begin{array}{l}\text { Human } \\
\text { rightness } \\
\text { score }\end{array}$ & $\begin{array}{l}\text { One-tailed } \\
\text { probability }\end{array}$ & $\begin{array}{l}\text { Two-tailed } \\
\text { probability }\end{array}$ \\
\hline $\mathrm{Ax} / \mathrm{I} / \mathrm{12}$ & 6 & 6 & 1.00 & 0.016 & 0.031 \\
$\mathrm{Ax} / \mathrm{III} / 33$ & 6 & 6 & 1.00 & 0.016 & 0.031 \\
$\mathrm{Ax} / \mathrm{III} / 68$ & 2 & 2 & 1.00 & 0.25 & 0.50 \\
$\mathrm{Ax} / \mathrm{III} / 74$ & 1 & 1 & 1.00 & 0.50 & 1.00 \\
$\mathrm{Ax} / \mathrm{III} / 84$ & 1 & 1 & 1.00 & 0.50 & 1.00 \\
$\mathrm{Ax} / \mathrm{III} / 89$ & 3 & 3 & 1.00 & 0.13 & 0.25 \\
Ax/III/106 & 5 & 1 & 0.20 & 0.97 & 0.31 \\
Ax/all figures combined & 24 & 20 & 0.83 & 0.00077 & 0.0013 \\
Ax/figures combined & 19 & 19 & 1.00 & 0.0000019 & 0.0000038 \\
$\quad$ excluding the last figure & & & & & \\
Nielsen/1.2 & 11 & 11 & 1.00 & 0.00049 & 0.00098 \\
\hline
\end{tabular}

The left-right ordering of taxa in the cladograms is strongly biased in favour of Homo sapiens

The human attention score was marginally significant in Animal evolution, the text that used one cladogram. In Das System der Metazoa, results differed among different cladograms, two out of seven being significantly anthropocentric. The overall results for this book depended on whether or not the different cladograms were weighted by their number of tips. The unweighted score was not significant, while the weighted score was. The findings are thus somewhat ambiguous. As weighting by numbers of tips is probably not justified in this case, one can conclude that Ax, overall, was able to avoid this aspect of anthropocentricism.

The human rightness score, on the other hand, was highly significant in both textbooks $(p<0.001)$, indicating that the position of man was indeed not randomly chosen. The taxon containing our species was systematically placed at the rightmost position in all cladograms but one. The only exception was Ax's figure III/106, which was adopted from another publication without changing the topology of the original cladogram (Waddell et al. 1999). Using two-tailed tests (i.e., estimating human "utterness" rather than "rightness") did not change the picture: although the probabilities were modified, significance levels remained largely unaffected.

\section{Discussion: why anthropocentricism matters}

The past 150 years have seen a gradual reduction in the anthropocentricism in biological systematics. Darwin (1859) removed the basis of beliefs that evolution is a directed process, and that Homo sapiens was the goal of evolution (or creation). The Darwinian mode of evolution is also incompatible with the idea that extant species can stand in ancestor-descendant relations to each other. Instead, extant species share common ancestors, from which they all descend. At this stage, the two first narrative devices lost their justification. Of course, this did not prevent them from turning up in a variety of scientific accounts through the decades (e.g., Romer 
1959). By now, however, such distortions of evolutionary history are largely restricted to the popular domain.

The second milestone was the "cladistic revolution" (Halstead 1978), started by Hennig (1950). His "phylogenetic systematics" identified monophyly as a desirable property of systematic entities. By doing so, Hennig removed human opinion and authoritative decisions from evolutionary biology. The phylogenetic (or cladistic) way of systemising threw out the arbitrariness of classification and replaced it by the scientific rigor of phylogenetics. It might not be intuitively clear why this should be relevant for anthropocentricism. The relevance lies in the fact that there is not a single paraphyletic taxon that incorporates the human species. On the other hand, quite many paraphyletic groupings denote taxa that would have been monophyletic if they had not been erected to the exclusion of a taxon containing man. Obviously, one unwritten rule for the erection of paraphyla was that they must not contain Homo sapiens. Examples of such paraphyla are "Pongidae" (= Hominidae sensu lato less Homo), "Prosimii" (= Primates less Anthropoidea), "Marsupionta" (= Mammalia less Placentalia), "Reptilia" (= Amniota less Aves and [crown]Mammalia), "Anamnia" (Vertebrata less Amniota), "Osteichthyes" (= Osteognathostomata less Tetrapoda), "Invertebrata" (Metazoa less Vertebrata), "Coelenterata" (Eumetazoa less Bilateria), "Protoctista" (Eukaryota less multicellular organisms), "Prokaryota" (Vita less Eukaryota). Inventing paraphyletic taxa that include our species may be a useful and funny exercise. Such taxa, like O'Hara's (1992, p. 154) "Anarthropoda", are so obviously absurd that no evolutionary taxonomist would ever have adopted them, no matter how large the phenetic gap between two sister taxa. When the phenetic gap that isolates tetrapods from lungfishes is large enough to justify the erection of a paraphyletic "Osteichthyes" - why doesn't the same apply to other taxa with many and very distinct autapomorphies, like the echinoderms and cnidarians? However, I suspect it is unthinkable even for evolutionary taxonomists to subdivide the Bilateria into the two superphyla "Anechinodermata" (= Chordata + Enteropneusta + Pterobranchia + Protostomia + Acoelomorpha) and Echinodermata, or to subdivide the Metazoa into the two subkingdoms "Acnidaria" (= Bilateria + Ctenophora + Porifera + Trichoplax adhaerens) and Cnidaria.

One might have thought that with the acceptance of Darwinian evolution and cladistic methodology, anthropocentricism was finally banned from evolutionary narratives, at least in scientific publications. This was the background for limiting my case study to textbooks that explicitly adopted a cladistic perspective. As one aim of phylogenetic systematists is to present phylogenetic history in a way that is independent of the opinion of authorities, one might expect that they are more conscious about avoiding anthropocentricisms than the average author of evolutionary textbooks. However, as my results show, this is merely wishful thinking. Two distorting devices can still occur even in state-of-the-art phylogenetic cladograms, and at least one of them is prevalent in textbooks: the taxon containing Homo sapiens is quite consistently placed at the rightmost position of each cladogram. There was also a trend that the resolution of cladogram branches is biased in favour of our own species $(p<0.10)$. 
These two narrative devices-i.e., O'Hara's fourth one and the one proposed by me-can be illustrated with Fig. 2. All trees in this figure are cladograms of the same taxon, viz. jawed vertebrates (Gnathostomata). Figure 2a is perfectly balanced, thus having eliminated device no. 4, but the taxon containing man (Choanata) is placed at the right, thus using device no. 5. The balance of the tree gives as much emphasis to "side branches" as to the branch leading to Homo sapiens and other Choanata. On the other hand, this branch is emphasized more than the others by placing it in the upper right corner, thus implying that one "has to read the graph" towards Choanata.

Figure $2 \mathrm{~b}$ is attempting to distribute taxa along the left-right axis in an objective way, thus eliminating device no. 5, but it is extremely unbalanced, thus using device no. 4. Every taxon's relative place was determined according to the number of described extant species within the taxon. This places the taxon containing man, Theria, somewhere in the middle of the tree. On the other hand, all taxa not including man are collapsed into only one single terminal taxon, resulting in a perfectly comb-like tree topology, again at the disadvantage of non-human taxa. Of course, it is possible to combine the advantages of both cladograms in one figure, just turning some branches of Fig. 2a around their own axes, the result of which is shown in Fig. 2c.

Figure $2 \mathrm{~d}$ shows a fourth out of the over 36,000 possibilities to depict gnathostome phylogeny using 8 tips. In this last illustration, the cladogram is maximally unbalanced, but this time to the advantage of the beluga or European
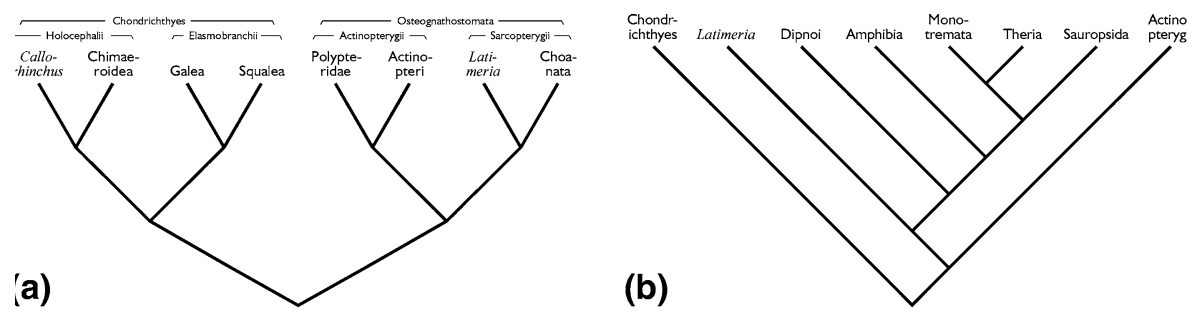

(c)
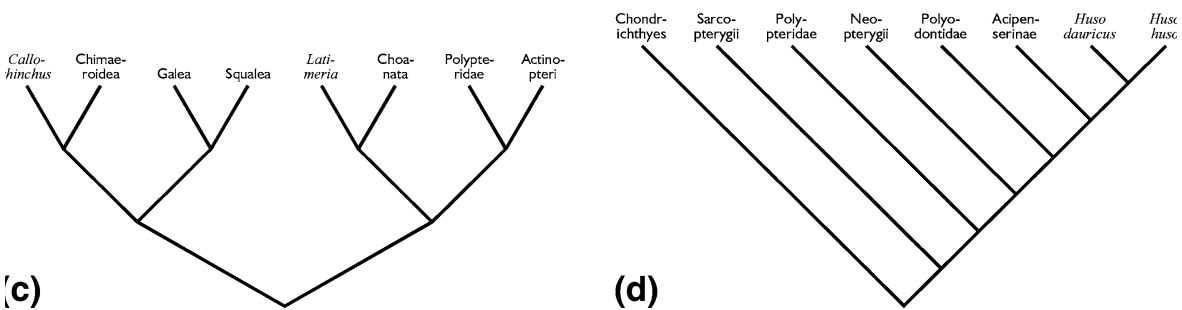

Fig. 2 Four out of more than 36,000 possibilities to represent gnathostome phylogeny using eight tips. See text for further explanations. (English vernacular names, where existent: Acipenserinae $=$ true sturgeons, Actinopteri $=$ true ray-finned fishes, Actinopterygii $=$ ray-finned fishes, Callorhinchus $=$ elephantfishes, Chondrichthyes $=$ cartilaginous fishes, Dipnoi $=$ lungfishes, Elasmobranchii $=$ sharks and rays, Holocephalii $=$ chimaeras, Huso dauricus $=$ kaluga, Huso huso = beluga, Latimeria $=$ coelacanths, Osteognathostomata $=$ bony $\quad$ vertebrates, $\quad$ Polyodontidae $=$ paddlefishes, $\quad$ Polypteridae $=$ bichirs, Sauropsida $=$ birds and reptiles, Theria $=$ true [viviparous] mammals.) 
Fig. 3 Phylogeny of gnathostomes using a maximally balanced representation and resolving up to seven bifurcations per branch. The phylogeny follows Sandvik (2001) and references therein. The sorting criterion for taxa along the vertical axis is their species number (see text)

sturgeon (Huso huso) and to the disadvantage of Sarcopterygii (including Homo sapiens) and five other side branches. Such presentations are quite uncommon, even though ichthyocentric cladograms are as justified as anthropocentric cladograms (but see Scott (1986) for a papiliocentric tree of life).

Finally, Fig. 3 shows an attempt of displaying the phylogeny of Gnathostomata as balanced as possible, resolving all branches to the same degree as the therian branch in Fig. 2a. Figure 3 also illustrates that there are natural constraints to perfectly balanced trees: the tree of life is not itself perfectly balanced (Guyer and Slowinski 1991; Mooers 1995; Mooers and Heard 2002), and several of the branches in Fig. 3 cannot be shown with higher resolution because they represent single species. This is why this tree does not have $2^{7}=128$ tips but merely 76 .

One might argue that the use of these narrative devices has certain advantages, i.e., that anthropocentric cladograms are not only negative. As regards device no. 4, using balanced trees such as Fig. 3 needs far more space for representing the same bunch of the tree than comb-like trees such as Fig. 2b. This depends of course on the aim of the cladogram. When one wants to tell the history of man (or any other taxon), and nothing else, comb-like trees are, of course, perfectly suited. However, in zoology textbooks this is normally not the intention, instead the reader is expected to get an impression of zoological diversity. This may be better achieved by using balanced trees. What is otherwise suggested is that there is no "relevant" or "interesting" diversity in "side branches".

It might also be argued that, because the tree of life itself is not balanced (cf. Fig. 3), cladogram balance is not a desirable attribute in the first place. In most cases, however, cladogram resolution is not constrained by the topology of the tree of life. Moreover, even in the cases where it is, it may be very useful heuristically to visualise that some tips in fact are species or species-poor taxa. This makes readers of the cladogram understand that biodiversity is indeed not distributed uniformly across the tree of life (as implicitly suggested by the straitjacket of Linnean categories, see below).

A further problem of balanced trees, also pointed out by O'Hara (1992, p. 150f), is that there are certain well-known taxon names and that these are not equally distributed among taxa with the same rank. ${ }^{2}$ As illustrated by Fig. 2a, very few of the names appearing at the tips of the cladogram will be known to a reader who is not already familiar with vertebrate phylogeny. That Choanata includes lungfishes (Dipnoi) and terrestrial vertebrates (Tetrapoda) is only known to specialists (many of whom would not even use the scientific name Choanata, but prefer other names

\footnotetext{
${ }^{2}$ By rank I refer to a relative measure of a taxon's inclusiveness (Ax 1995-2001). It can be envisaged as being inversely proportional to the number of nodes between a taxon and the root of the tree. This measure is relative in such a way that sister taxa always have the same rank, while subordinated (superordinated) taxa have lower (higher) rank. Linnean categories are sometimes referred to as ranks, too, but those two concepts are unrelated.
} 


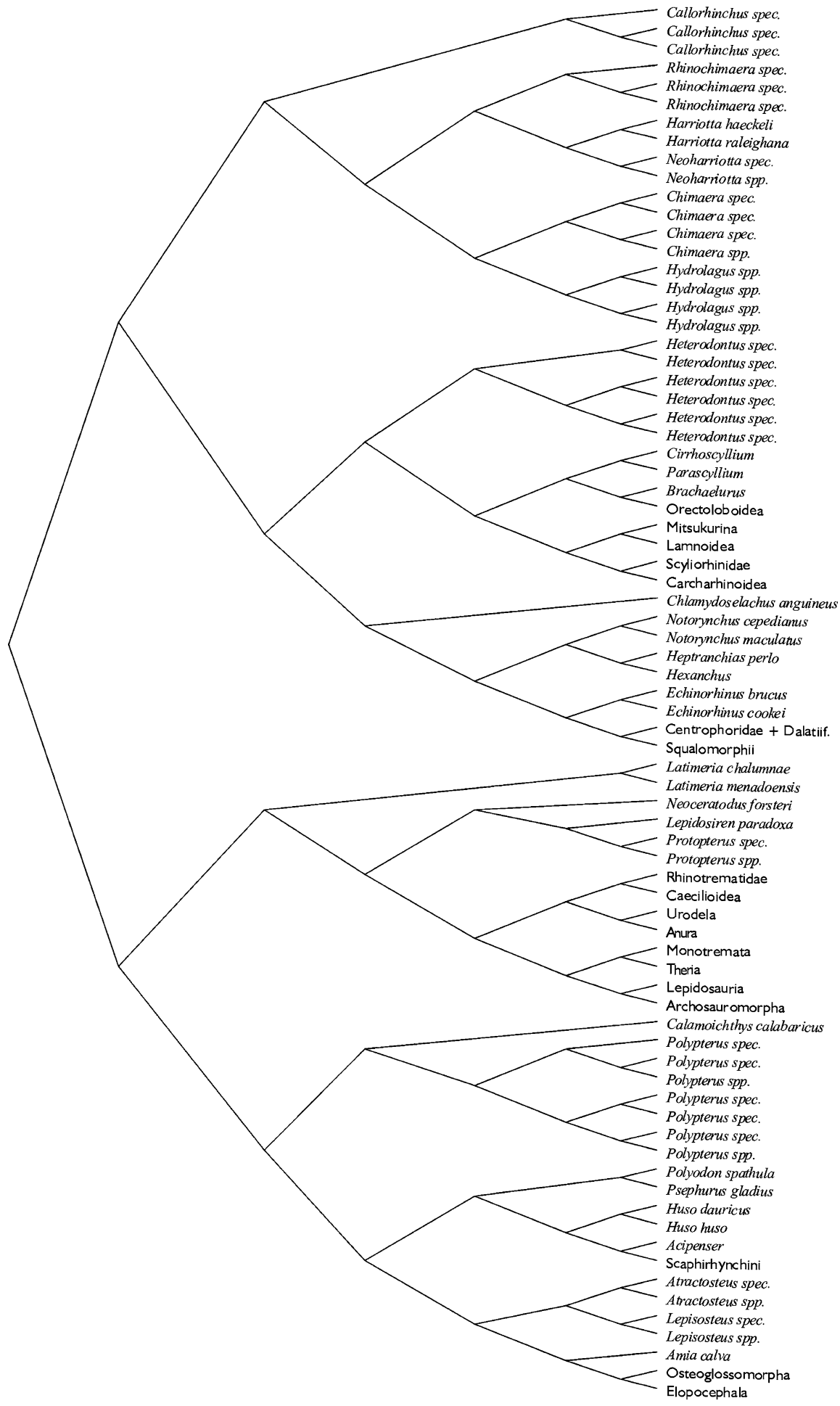


such as Rhipidistia sensu lato). Likewise, that Callorhinchus and Chimaeroidea together constitute the chimaeras (Holocephalii), or that Galea and Squalea together represent the rays and sharks (Elasmobranchii), will not be visible from the cladogram, unless names of higher taxa are displayed above the tips (as is shown in Fig. 2a, but not Fig. 2b-d). In such cases, perfect tree balance does not necessarily seem to outweigh the loss of information. A compromise would be to display one more branching in the rightmost branch of Fig. 2a, and to add names of higher taxa above the names of the tips.

As regards device no. 5, also the anthropocentric left-right orientation has certain advantages, not the least a better orientation of untrained biologists in the tree. However, this argument seems to be mostly a question of habit (and practice), as it also could be applied to device no. 3. (In fact it has been applied, by claiming that evolutionary systematics is more in accord with commonsense than phylogenetic systematics; Halstead 1978; Mayr and Bock 2002.) After all, taxa have to be sorted in one way or another, and as all sorting criteria are equally correct, it does not really seem to matter whether the criterion chosen is the phylogenetic distance to Homo sapiens.

What, then, are the disadvantages of anthropocentric cladograms, and do they weigh more than the advantages? Evidence that anthropocentric cladograms are indeed problematic comes from student questionnaires (O'Hara 1997; H. Sandvik, unpublished manuscript). If students are asked to draw the tree of life, they generally produce drawings that place Homo sapiens in a prominent position (either on top of the drawing or on its right-hand side). This in itself does not tell us whether the choice to draw a tree in this way is based on misconceptions about evolutionary processes, or just a matter of habit. However, there is also overwhelming evidence that many students are unable to read cladograms: even many graduate students of biology pay more attention to the left-right ordering of taxon names on the cladograms tips, than to the topology of the cladogram displayed beneath the taxon names.

A further problem is that students often regard Linnean categories (i.e., labels such as "family", "subphylum", "order" etc.) as scientific statements. They assume that the different levels in the Linnean hierarchy have distinct meanings and that the assignment of a given category to any one taxon is either right or wrong. Of course, Linnean categories are entirely arbitrary and do not carry any information whatsoever (de Queiroz and Gauthier 1992; Ereshefsky 1994, 2001, 2002; Donoghue 2001). This misconception is further reinforced by the fact that many textbook authors tend to adjust the resolution of cladograms to Linnean categories (for a refreshing exception, see Ax 1995-2001). In other words, taxa that got Linnean categories attached to them are overrepresented among the tips of cladograms. In some textbooks, this is even aggravated by the pruning of lessknown (and supposedly less important) branches (i.e., O'Hara's second narrative device). Cladograms therefore often only display the taxon names that the students are supposed to memorise, which is a somewhat biased sample of the taxa that in fact exist in nature.

These problems re-enforce each other in misleading at least untrained readers of cladograms. To be sure, none of those problems is solved by avoiding 
anthropocentricism in cladograms alone. It is at least as important to teach students how to read cladograms (O'Hara 1997; Sandvik, unpublished manuscript). O'Hara (1992, p. 156) emphasized that it "should always be made clear in a tree diagram that it is the topological relations of the branches that carries meaning, and not their left-to-right positioning". However, stating this point somewhere in the vicinity of a tree diagram does not prevent the readers of the diagram from unconsciously noticing the implicit sequencing of taxa. As graphs appeal to the optical memory, the right taxon will almost necessarily be remembered as the "focal" one, even if the accompanying text tells the reader otherwise. In addition to increased awareness about the fact that tree thinking is an ability that has to be acquired, authors and teachers should become more aware of how they present cladograms. It they are not, it turns out that more or less unconsciously the result becomes anthropocentrically biased.

This seems to suggest that other modes to determine taxa positions along the leftright axis should be considered by authors and teachers. Several such alternatives to the anthropocentric mode have been proposed. de Queiroz and Gauthier (1992) suggested to sort taxa according to the number of species contained in them: at each node, the taxon encompassing most species-either described or estimated, excluding or including fossil ones-is placed to the right of the smaller taxon (cf. Figs. 2b, c and 3). Secondly, several authors seem to use a kind of implicit complexity measure: at each node, the taxon having acquired more, or more complex, autapomorphies is placed to the right of the taxon that has undergone less evolutionary change. This is evidently how e.g., Ax (1995-2001) has ordered taxa in cladograms that do not contain Homo sapiens-even though this criterion is repeatedly violated in cladograms that $d o$ contain us. A third alternative, which has been carried through in a Norwegian textbook of mine (Sandvik 2001), is alphabetic order: at each node the two taxa are ordered simply by their scientific names. There are advantages and disadvantages to all those methods. The latter one is deliberately arbitrary. It is perhaps best suited to illustrate that left-right order does indeed not carry any meaning whatsoever. The apomorphy-based method is somewhat more in accordance with intuition. However, it accepts the horizontal orientation of a cladogram as a narrative axis, at which most change occurs. This, and the fact that there is no objective measure of the complexity of autapomorphous (or any other) traits (Johnson 1968; Ghiselin 1969; Griffiths 1973), suggests that this method has its weaknesses. The method based on species numbers might be the best in order to express the importance of taxa in terms of biodiversity. As such, this method might be recommended for most uses.

All three methods are probably better than leaving the left-right ordering to "chance", since the result is not random at all, but anthropocentrically biased. This is most likely explainable by an innate psychological bias to think anthropocentrically. If this disposition is not kept in check by conscious decisions, it obviously introduces systematic biases in how we choose to represent evolutionary history. These findings do of course not relate to the validity of the scientific results displayed by the cladograms. To the contrary, it is exactly because the graphical representation of the results is irrelevant to their correctness that scientists are entirely free to choose whatever resolution and ordering of taxa they like. Only 
because of this freedom, unconscious biases can affect the graphical result in the way they do and may thereby reveal some underlying expectations or worldviews of the scientists. Given that textbook authors-particularly cladistic ones-should be expected to be especially aware of issues that can confuse or mislead readers, this subconscious bias seems to be strong indeed.

In conclusion, the cladistic revolution has indeed largely removed three of the narrative devices that distort accounts of evolutionary history. However, two devices can also be found in cladistic depictions of phylogenetic relationships, viz. differential resolution of branches and left-right ordering. The latter narrative device has been found to be present even in textbooks that explicitly adopt a cladistic perspective. O'Hara (1992, pp. 154-156) has provided a very useful guide on how to "tell the tree", and authors of textbooks and of original papers presenting phylogenetic research would profit from following those suggestions. The current paper illustrates that having the right intentions does not suffice to produce unbiased evolutionary narratives. Obviously, the subconsciousness even of trained cladists is more anthropocentric than we would like to acknowledge.

Open Access This article is distributed under the terms of the Creative Commons Attribution Noncommercial License which permits any noncommercial use, distribution, and reproduction in any medium, provided the original author(s) and source are credited.

\section{References}

Ax P (1995-2001) Das System der Metazoa. Ein Lehrbuch der phylogenetischen Systematik, 3 volumes, Fischer, Stuttgart, and Spektrum, Heidelberg (English translation, 1996-2003, Multicellular animals, Springer, Berlin)

Darwin C (1859) On the origin of species by means of natural selection, or the preservation of favoured races in the struggle for life. Murray, London

de Queiroz K, Gauthier J (1992) Phylogenetic taxonomy. Annu Rev Ecol Syst 23:449-480

Donoghue MJ (2001) A wish list for Systematic Biology. Syst Biol 50:755-757

Ereshefsky M (1994) Some problems with the Linnean hierarchy. Philos Sci 61:186-205

Ereshefsky M (2001) The poverty of the Linnean hierarchy: a philosophical study of biological taxonomy, Cambridge Studies in Philosophy and Biology, 15 (ed. M. Ruse). Cambridge University Press, Cambridge

Ereshefsky M (2002) Linnean ranks: vestiges of a bygone era. In: Barrett JA, Alexander JMcK (eds) PSA 2000: proceedings of the 2000 biennial meeting of the philosophy of science association. Part II: symposia papers. Philosophy of Science Association, East Lansing, Michigan, pp S305-S315

Farris JS (1976) Expected asymmetry of phylogenetic trees. Syst Zool 25:196-198

Felsenstein J (2004) Inferring phylogenies. Sinauer, Sunderland

Ghiselin MT (1969) The principles and concepts of systematic biology. In: Sibley CG, Alexander RD, Constance L, Corliss JO, Cowan RS, Lockhart WR, Mayr E, Wagner WH Jr (eds) Systematic biology. National Academy of Sciences, Washington, DC, pp 45-66

Griffiths GCD (1973) Some fundamental problems in biological classification. Syst Zool 22:338-343

Guyer C, Slowinski JB (1991) Comparisons of observed phylogenetic topologies with null expectations among three monophyletic lineages. Evolution 45:340-350

Halstead LB (1978) The cladistic revolution - can it make the grade? Nature (Lond) 276:759-760

Hennig W (1950) Grundzüge einer Theorie der Phylogenetischen Systematik. Deutscher Zentralverlag, Berlin

Johnson LAS (1968) Rainbow's end: the quest for an optimal taxonomy. Proc Linn Soc N S W 93:1-45 [reprinted, 1970, Syst Zool 19:203-39] 
Kirkpatrick M, Slatkin M (1993) Searching for evolutionary patterns in the shape of a phylogenetic tree. Evolution 47:1171-1181

Mayr E, Bock WJ (2002) Classifications and other ordering systems. J Zool Syst Evol Res 40:169-194 Mooers AØ (1995) Tree balance and tree completeness. Evolution 49:379-384

Mooers AØ, Heard SB (2002) Developing uses for phylogenetic tree shape in the study of evolution. Syst Biol 51:833-929

Nielsen C (2001) Animal evolution: interrelationships of the living phyla, 2nd edn. Oxford University Press, Oxford

O'Hara RJ (1992) Telling the tree: narrative representation and the study of evolutionary history. Biol Philos 7:135-160

O'Hara RJ (1997) Population thinking and tree thinking in systematics. Zool Scr 26:323-329

$\mathbf{R}$ development core team (2005) R: a language and environment for statistical computing, version 2.1.1, URL: http://www.r-project.org, R Foundation for Statistical Computing, Wien

Romer AS (1959) The vertebrate story, 4th edn. Chicago University Press, Chicago

Sandvik H (2001) Dyrenes evolusjon - en innføring i systematisk zoologi og dyrenes stamtre. Tapir, Trondheim

Scott JA (1986) The butterflies of North America: a natural history and field guide. Stanford University Press, Stanford

Slowinski JB (1990) Probabilities of n-trees under two models: a demonstration that asymmetrical interior nodes are not improbable. Syst Zool 39:89-94

Waddell PJ, Okada N, Hasegawa M (1999) Towards resolving the interordinal relationships of placental mammals. Syst Biol 48:1-5 